\title{
Design of Compact Polarization Insensitive Triple Bandstop Frequency Selective Surface with High Stability under Oblique Incidence
}

\author{
Anumoy GHOSH ${ }^{1}$, Amartya BANERJEE ${ }^{2}$, Santanu DAS ${ }^{3}$ \\ ${ }^{1}$ Dept. of Electronics \& Communication Engineering, National Institute of Technology, Mizoram, Aizawl, India \\ ${ }^{2}$ Dept. of Electronics \& Telecommunication Engineering, Jadavpur University, Kolkata, India \\ ${ }^{3}$ Dept. of Electronics \& Telecommunication Engineering, Indian Inst. of Eng. Science \& Technology, Shibpur, India
}

anumoy.science@gmail.com, amartyapotter@gmail.com, santanumdas@yahoo.com

Submitted December 22, 2018 / Accepted June 5, 2019

\begin{abstract}
This paper proposes a novel design of a triple band frequency selective surface (FSS) that acts as a bandstop filter at $1.92 \mathrm{GHz}, 3.5 \mathrm{GHz}$ and $5.64 \mathrm{GHz}$. The bandstop frequencies resemble to GSM, WLAN and WiMAX application bands respectively. The structure is polarization insensitive since its frequency response remains unaltered for both TE and TM modes of electromagnetic wave propagation. The main attractive feature of the structure is its highly stable response under oblique incident angle up to $\pm 80^{\circ}$ at both TE and TM polarizations. Surface current distribution and equivalent circuit modeling are presented to demonstrate the resonance characteristic of the FSS. The structure is compact with an overall unit cell area of $0.09 \lambda \times 0.09 \lambda$, where $\lambda$ is the wavelength corresponding to the lowest resonant frequency. The proposed structure is compared with other multi-bandstop FSS structures in literature to highlight its superior functionality in terms of stability under oblique incidence and compactness. A prototype comprising of $6 \times 6$ array of the proposed unit cell is fabricated and the measured results are in good agreement with the simulated results.
\end{abstract}

\section{Keywords}

Frequency selective surface, bandstop filter, polarization insensitive, oblique incidence angle, compact

\section{Introduction}

Frequency selective surfaces (FSS) have attracted the attention of researchers for their variety of electromagnetic (EM) properties which include their use as bandstop and bandpass filters [1], [2], artificial magnetic conductors [3], polarization converters [4] and reactive impedance surfaces [5]. The FSS structures exhibiting bandstop filter characteristics find applications in EM shielding, antenna beam shaping and reflector antennas [6-8]. Since, EM waves are subject to multipath propagation and FSS structures cannot be always put perpendicular to the incident EM waves, stable and unperturbed response is necessary for bandstop FSS under oblique incidence. Moreover, due to the randomness in EM environment, polarization insensitivity is also required. For applications like EM shielding, it is desirable that a single FSS structure should be capable of inhibiting interference from multiple wireless frequencies. In order to satisfy these requirements, multiple bandstop FSS with polarization insensitivity and stability under oblique incidence are seeking attention and have been reported in recent literature [9-16]. However, for FSS structures acting as multiple bandstop filter (BSF), the response under oblique incidence gets worse as it approaches towards grazing angle of $\pm 90^{\circ}$. Hence, stable characteristic is limited to, generally, within $\pm 60^{\circ}$ incident angle in case of triple band FSS [12-16]. As a result, the use of multiband bandstop FSS becomes limited in complex multipath environment.

The proposed work addresses the issue of unstable performance under oblique incidence as the incident angle approaches $\pm 90^{\circ}$. The proposed FSS unit cell consists of three metallic structures, two at its top surface and one at its bottom surface, which provide triple bandstop resonances at GSM, WLAN and WiMAX frequencies. Proper justifications regarding the generation of stop bands by investigating the current distribution patterns and equivalent circuit modeling are presented. The metallic structures are made to be rotational symmetric to ensure polarization insensitivity. Closed loop structures with meandered arms are designed to obtain stability at oblique angle of incidence and achieve compactness. The proposed design is compared with other previously reported bandstop FSS structures to demonstrate its superior performance under oblique incidence thus resolving the limitation of using multi-bandstop FSS in varied multipath environment. All the simulations pertaining to the proposed work are carried out using FEM based simulation software ANSYS HFSS v. 15. 


\section{FSS Geometry}

The FSS unit cell comprises of FR4 substrate with dimensions $14 \mathrm{~mm} \times 14 \mathrm{~mm} \times 1.6 \mathrm{~mm}$. The substrate has dielectric constant of 4.4 and loss tangent 0.02 . Figures 1(a) and 1(b) depict the configurations of the top and bottom surfaces of the FSS unit cell respectively. The relevant dimensions are $\mathrm{A} 1=4.7 \mathrm{~mm}, \mathrm{~A} 2=3.2 \mathrm{~mm}, \mathrm{~A} 3=1.05 \mathrm{~mm}$, $\mathrm{A} 4=1.45 \mathrm{~mm}, \mathrm{~A} 5=1.5 \mathrm{~mm}, \mathrm{~B} 1=2.1 \mathrm{~mm}, \mathrm{~B} 2=1.4 \mathrm{~mm}$, $\mathrm{B} 3=2.7 \mathrm{~mm}, \mathrm{C} 1=1.3 \mathrm{~mm}, \mathrm{C} 2=4.05 \mathrm{~mm}, \mathrm{C} 3=0.4 \mathrm{~mm}$, $\mathrm{G} 1=0.3 \mathrm{~mm}, \quad \mathrm{G} 2=0.25 \mathrm{~mm}, \quad \mathrm{D} 1=3.45 \mathrm{~mm}, \quad \mathrm{D} 2=$ $1.5 \mathrm{~mm}, \mathrm{D} 3=1.6 \mathrm{~mm}, \mathrm{D} 4=2.3 \mathrm{~mm}, \mathrm{D} 5=3.75 \mathrm{~mm}, \mathrm{G} 3=$ $0.6 \mathrm{~mm}$ and $\mathrm{G} 4=0.25 \mathrm{~mm}$. All the metallic structures are square rings with meandered sides. Due to this geometry, the metallic structures exhibit rotational symmetry, that is, they have the same symmetry with respect to both X and $\mathrm{Y}$ axes. For simulation purpose, the unit cell is kept in a box with appropriate master-slave boundary conditions to make an infinite array of the unit cell in both the $\mathrm{X}$ and $\mathrm{Y}$ axes. The excitation ports are assigned Floquet Port properties.

Figure 2 illustrates the step-wise evolution of the geometry of the FSS unit cell from simple square rings to meander-armed square rings. The transmission performances of the designs are depicted in Fig. 3 which reflects that the progressive meandering of the square rings leads to gradual decrease in the resonance frequencies due to increase in the current path.

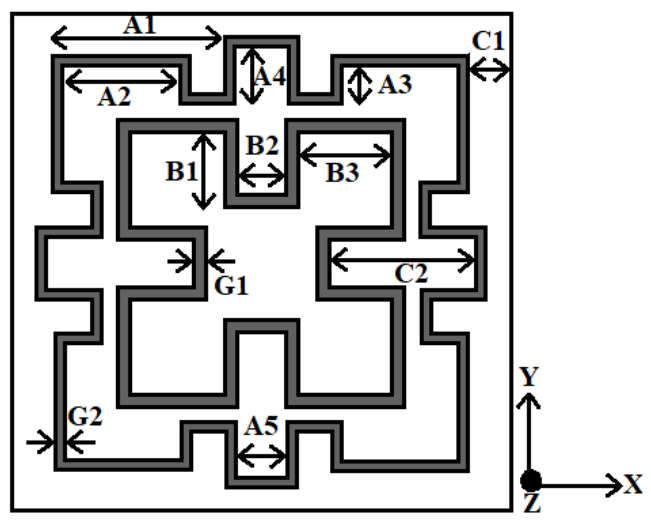

(a)

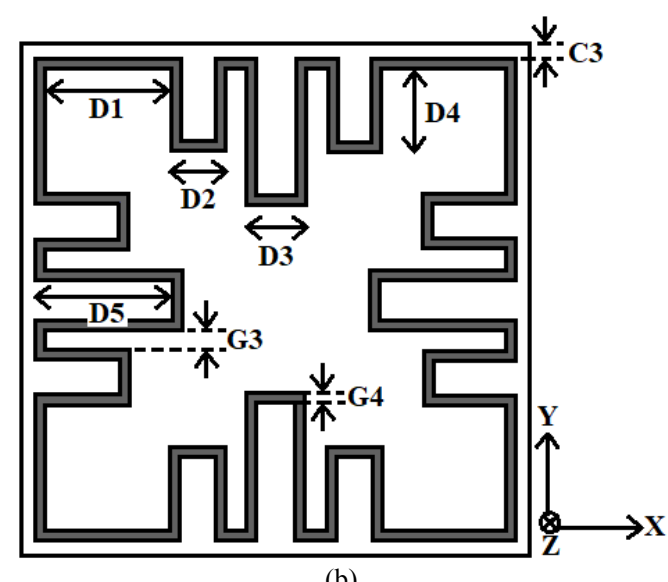

(b)

Fig. 1. Configuration of FSS unit cell: (a) Top view. (b) Bottom view.

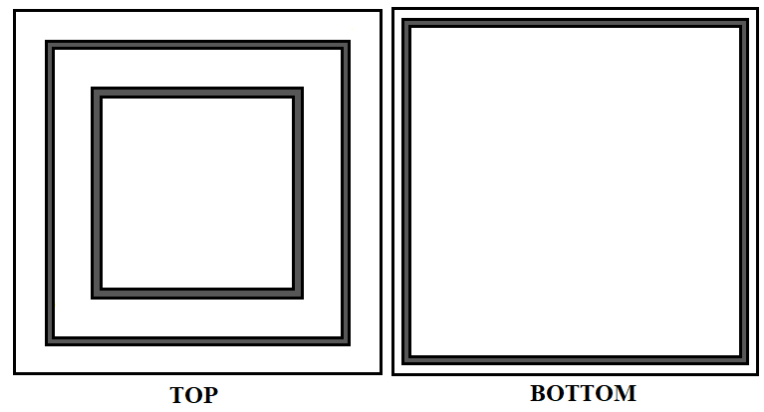

(a)

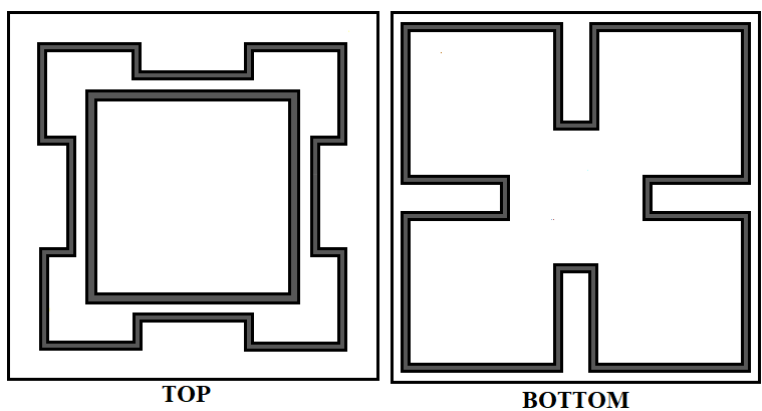

(b)

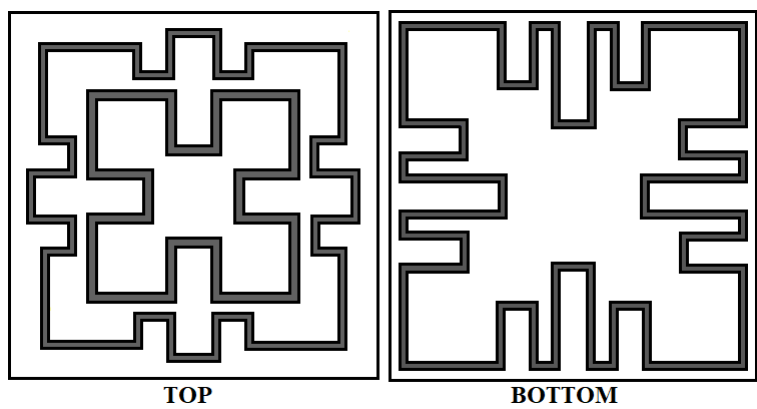

(c)

Fig. 2. Evolution of FSS geometry from simple square rings to meander-armed square rings: (a) FSS 1; (b) FSS 2; (c) FSS 3 (proposed).

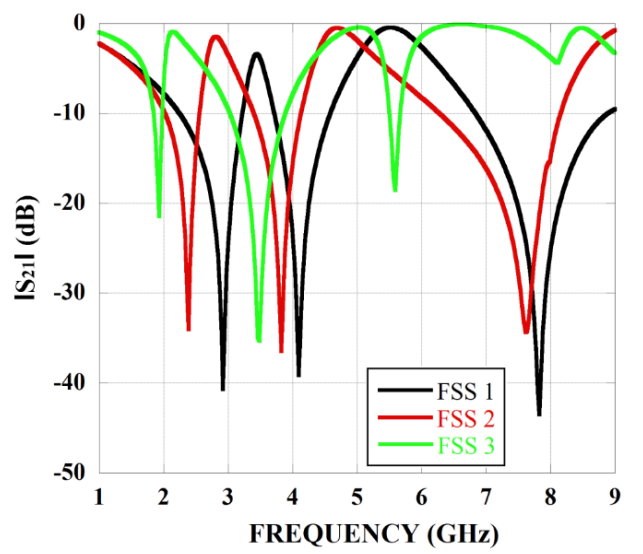

Fig. 3. Transmission characteristic of the various FSS unit cells as presented in Fig. 2.

\section{Results and Discussions}

Figure 4 shows the $\left|\mathrm{S}_{21}\right|$ response of the proposed FSS under normal incidence for both TE and TM modes of 
propagating waves. From the graph, it is observed that the FSS acts as a triple bandstop filter at $1.92 \mathrm{GHz}$ (corresponds to GSM frequency) with $7.25 \%$ fractional bandwidth (FBW), at $3.5 \mathrm{GHz}$ (corresponds to WLAN frequency) with $24.06 \% \mathrm{FBW}$ and at $5.64 \mathrm{GHz}$ (corresponds to WiMAX frequency) with $3.22 \%$ FBW. FBW is calculated by considering $\left|\mathrm{S}_{21}\right| \leq-10 \mathrm{~dB}$. The figure also highlights that the $\left|\mathrm{S}_{21}\right|$ response of the structure remains unperturbed for both the TE and TM modes thus confirming its polarization insensitivity which is achieved due to the rotational symmetry of the structure.

An FSS acting as bandstop filter should be immune to any oblique incidence of EM waves as in free space, it is highly unlikely that the orientation of the propagating waves will always be perpendicular to the plane of the FSS structure. Hence, the proposed structure is illuminated under oblique incidence and the response is presented in Figs. 5(a) and 5(b). From the figures, it is evident that $\left|S_{21}\right|$ characteristic of the structure remains almost undisturbed up to incident angle of $\pm 80^{\circ}$ which is very close to the grazing angle of $\pm 90^{\circ}$. Furthermore, the figures also highlight that for any angle of incidence, the response remains almost similar for both the TE and TM modes. Table 1 highlights the frequency values corresponding to each oblique incidence for both TE and TM modes.

In order to comprehend the novelty of the proposed structure, it is compared with previously reported bandstop

\begin{tabular}{|c|c|c|}
\hline \multirow{2}{*}{$\begin{array}{c}\text { Angle of } \\
\text { incidence }\end{array}$} & \multicolumn{2}{|c|}{ Resonant frequency (GHz) } \\
\cline { 2 - 3 } & TE mode & TM mode \\
\hline $0^{\circ}$ & $1.92,3.5,5.64$ & $1.92,3.48,5.68$ \\
\hline $\pm 20^{\circ}$ & $1.92,3.47,5.64$ & $1.92,3.48,5.68$ \\
\hline $\pm 40^{\circ}$ & $1.92,3.5,5.61$ & $1.92,3.48,5.68$ \\
\hline $\pm 60^{\circ}$ & $1.93,3.47,5.59$ & $1.92,3.5,5.68$ \\
\hline $\pm 80^{\circ}$ & $1.93,3.5,5.58$ & $1.92,3.5,5.65$ \\
\hline
\end{tabular}

Tab. 1. Resonance of the proposed FSS under oblique incidence in TE and TM modes.

\begin{tabular}{|c|c|c|c|c|}
\hline Ref. & Unit cell size & $\begin{array}{c}\text { No. of } \\
\text { stopbands }\end{array}$ & $\begin{array}{c}\text { Stability } \\
\text { under } \\
\text { oblique } \\
\text { incidence }\end{array}$ & $\begin{array}{c}\text { Maximum } \\
\text { frequency } \\
\text { deviation }\end{array}$ \\
\hline$[8]$ & $0.056 \lambda \times 0.056 \lambda$ & 1 & up to $\pm 60^{\circ}$ & $0.4 \%$ \\
\hline$[9]$ & $0.065 \lambda \times 0.065 \lambda$ & 2 & up to $\pm 75^{\circ}$ & $0.9 \%$ \\
\hline$[10]$ & $0.42 \lambda \times 0.42 \lambda$ & 2 & up to $\pm 45^{\circ}$ & $\begin{array}{c}\text { Data } \\
\text { unavailable }\end{array}$ \\
\hline$[11]$ & $0.11 \lambda \times 0.11 \lambda$ & 2 & up to $\pm 60^{\circ}$ & $2.9 \%$ \\
\hline$[12]$ & $0.13 \lambda \times 0.13 \lambda$ & 3 & up to $\pm 30^{\circ}$ & $\begin{array}{c}\text { Data } \\
\text { unavailable }\end{array}$ \\
\hline$[13]$ & $0.066 \lambda \times 0.066 \lambda$ & 3 & up to $\pm 60^{\circ}$ & $0.9 \%$ \\
\hline$[14]$ & $0.12 \lambda \times 0.12 \lambda$ & 3 & up to $\pm 35^{\circ}$ & $\begin{array}{c}\text { Data } \\
\text { unavailable }\end{array}$ \\
\hline$[15]$ & $0.15 \lambda \times 0.15 \lambda$ & 3 & up to $\pm 45^{\circ}$ & $0.9 \%$ \\
\hline$[16]$ & $0.1 \lambda \times 0.1 \lambda$ & 3 & up to $\pm 60^{\circ}$ & $3.2 \%$ \\
\hline Proposed & $0.09 \lambda \times 0.09 \lambda$ & 3 & up to $\pm 80^{\circ}$ & $1.1 \%$ \\
\hline
\end{tabular}

Tab. 2. Comparison of the proposed FSS with other bandstop FSS reported in literature.

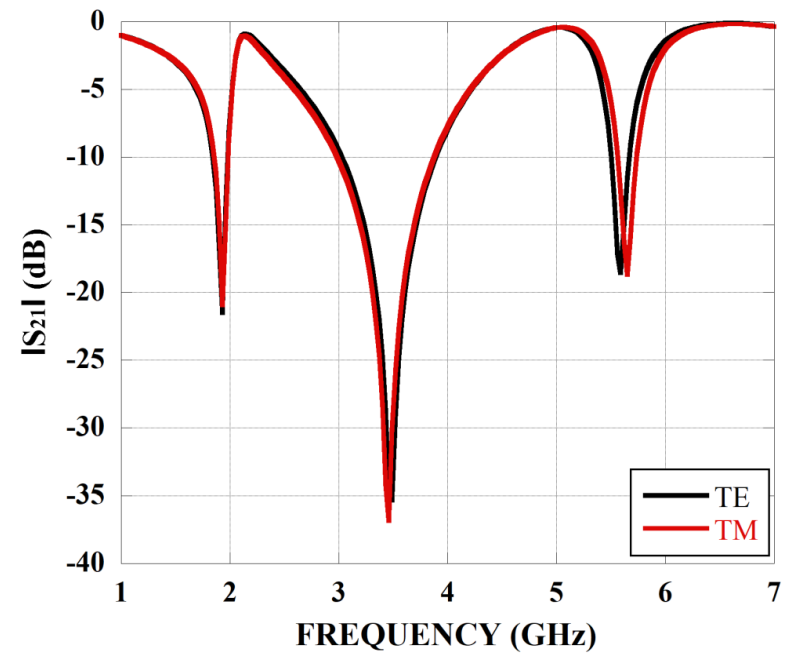

Fig. 4. Simulated $\left|S_{21}\right|$ response of the proposed FSS under normal incidence for both TE and TM polarizations.

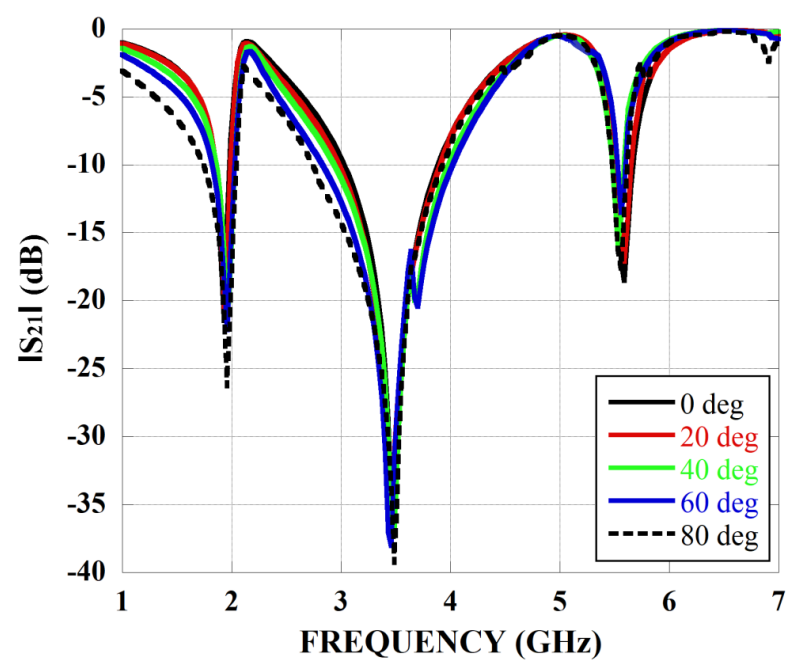

(a)

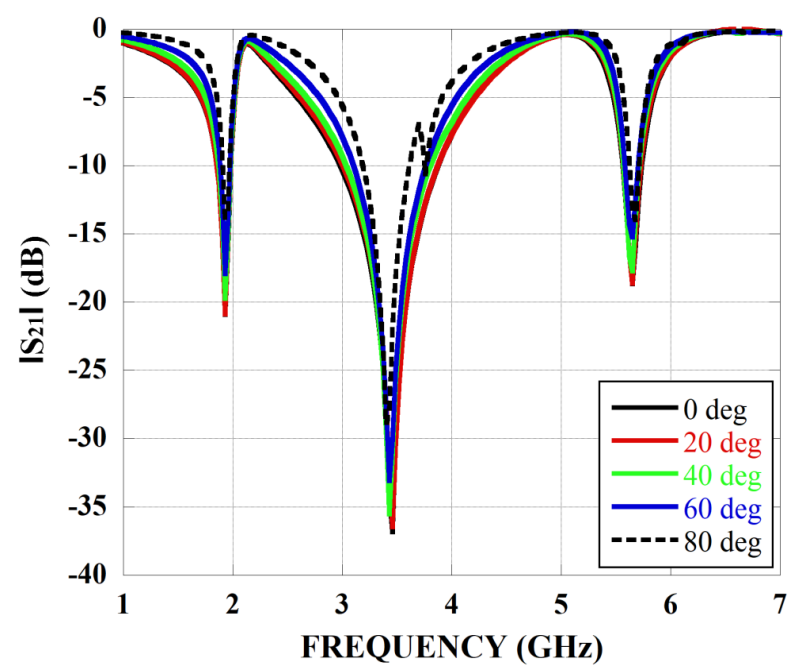

(b)

Fig. 5. Simulated $\left|S_{21}\right|$ response of the proposed FSS under oblique incidence: (a) TE mode; (b) TM mode.

FSS [8-16] and the results are tabulated in Tab. 2. The table highlights that for dual bandstop FSS [9-11], maximum stability under oblique incidence is provided by [9] up 
to $\pm 75^{\circ}$. However, when bandstop resonances increase to three, the maximum stability under oblique incidence is reduced to $\pm 60^{\circ}$ [13], [16]. Hence, triple bandstop FSS will fail to act as bandstop filter where they are exposed to complex multipath environment when incident electromagnetic wave may frequently approach $\pm 90^{\circ}$. As revealed in the table, the proposed FSS not only provides triple bandstop resonance but also it maintains stable performance under oblique incidence up to $\pm 80^{\circ}$ which is maximum of all the compared structures thus qualifying itself to be the most prominent candidate in terms of immunity towards oblique incidence. Compactness is another important feature since for a given area, maximum number of FSS unit cells are desired to be accommodated. In this context, the proposed FSS unit cell is more compact than most of the structures except [9], [13]. It is to be noted that the maximum frequency deviation for the proposed work is $1.1 \%$ which is noticed at the third resonance in TE mode. Although some previous works have better performance with respect to maximum frequency deviation, but they are unable to maintain stability under oblique incidence up to $\pm 80^{\circ}$. Furthermore, it is to be noted that if oblique incidence up to $\pm 60^{\circ}$ is taken into account, the proposed FSS shows maximum frequency deviation of $0.9 \%$ which is comparable to $[9,13,15]$.

\subsection{Current Distribution Analysis at the Notched Bands}

The surface current distributions at the stopband frequencies on the FSS unit cell are investigated and presented in Fig. 6. The current path indicated by a-b-c-d-e-f-g-h-i-j$\mathrm{k}-1-\mathrm{m}$ in Fig. 6(a) provides the first resonance. The paths denoted by n-o-p-q-r-s in Fig. 6(b) and t-u-v-w-x in Fig. 6(c) generate the second and third stopband resonances respectively. For each of the current paths, it is observed that current minima are formed at the ends and a current maxima is formed at the middle of the path thus indicating that it acts as a half-wavelength path for the corresponding stopband frequency. If the current path length is $L$, then the respective stopband frequency is given as,

$$
f_{\mathrm{S}}=\frac{c}{2 L \sqrt{\varepsilon_{\mathrm{eff}}}} .
$$

Here, $c$ is the speed of light in vacuum. $\varepsilon_{\text {eff }}$ is related to relative permittivity $\varepsilon_{\mathrm{r}}$, height of the substrate $h$ and width of metallic strips $w$ as [17],

$$
\varepsilon_{\text {eff }}=\frac{\varepsilon_{\mathrm{r}}+1}{2}+\frac{\varepsilon_{\mathrm{r}}-1}{2}\left(1+12 \frac{h}{w}\right)^{1 / 2} .
$$

\subsection{Impedance Analysis at the Notch Bands}

The equivalent circuit of the FSS unit cell is presented in Fig. 7. Each of the metallic loops is modelled as a series LC circuit [18]. Since, each metallic structure has two halfwavelength current paths, the inductor is further simplified to be a parallel combination of two identical inductors. $Z_{\mathrm{D}}$ indicates the intrinsic impedance of the dielectric substrate. The bandstop resonance due to each LC branch is given by,

$$
f_{\mathrm{S}}=\frac{1}{2 \pi \sqrt{0.5 L_{i} C_{i}}}, i=1,2,3 .
$$

The values of the lumped elements are derived using quasi-static analysis outlined in [9] and the evaluated values are: $\mathrm{L} 1=34.38 \mathrm{nH}, \mathrm{C} 1=0.12 \mathrm{pF}, \mathrm{L} 2=22.2 \mathrm{nH}$, $\mathrm{C} 2=0.07 \mathrm{pF}, \mathrm{L} 3=66.94 \mathrm{nH}$ and $\mathrm{C} 3=0.21 \mathrm{pf}$.

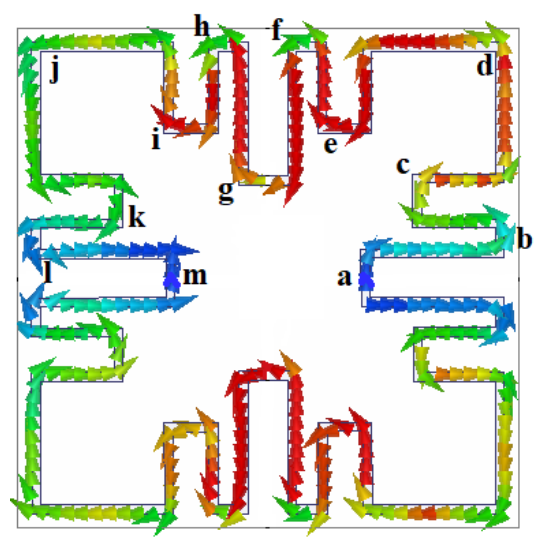

(a)

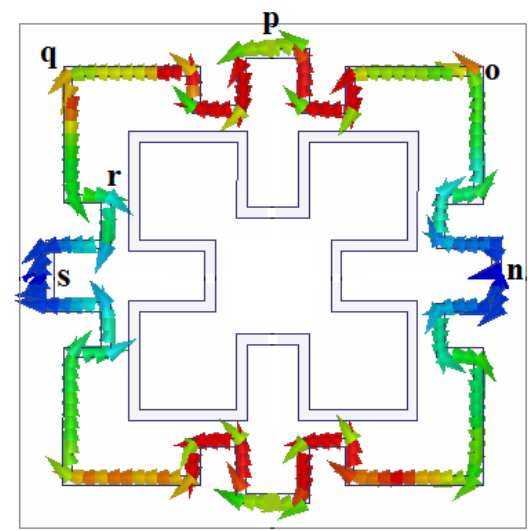

(b)

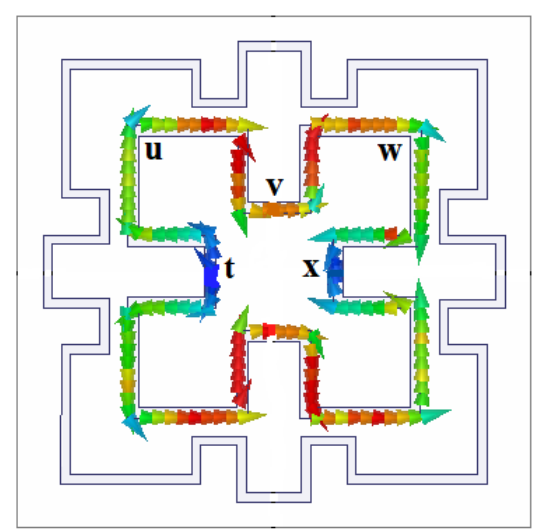

(c)

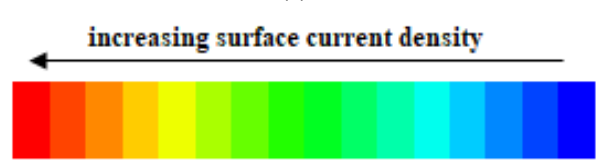

Fig. 6. Simulated surface current distribution on the proposed FSS unit cell at: (a) $1.9 \mathrm{GHz}$; (b) $3.6 \mathrm{GHz}$; (c) $5.4 \mathrm{GHz}$. 


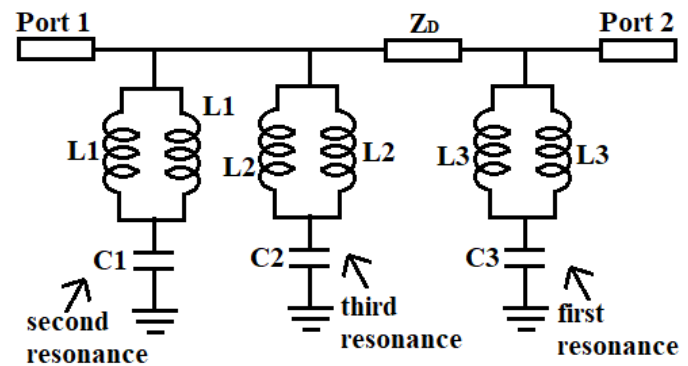

Fig. 7. Equivalent circuit of the proposed FSS unit cell.

\section{Fabrication and Measurement}

The simulation set-up assumes an infinite array of the proposed unit cell in two dimensions. To mimic this condition, a prototype is fabricated comprising of an array of $6 \times 6$ elements as presented in Fig. 8 .

The $\left|\mathrm{S}_{21}\right|$ response is measured using free-space measurement technique which consists of appropriate horn antennas, the fabricated structure mounted on a turn table for measurement under oblique incidence and a Vector Network Analyzer (VNA) of make Agilent PNA-L 5235A. The measurement set-up is depicted in Fig. 9. To ensure measurement accuracy, the $\left|S_{21}\right|$ response without the prototype is measured first. This data is used to calibrate the $\left|\mathrm{S}_{21}\right|$ response measured with the fabricated structure. The horn antennas are used in $\mathrm{E}$ and $\mathrm{H}$ planes for measurement under TE and TM modes of excitations respectively.

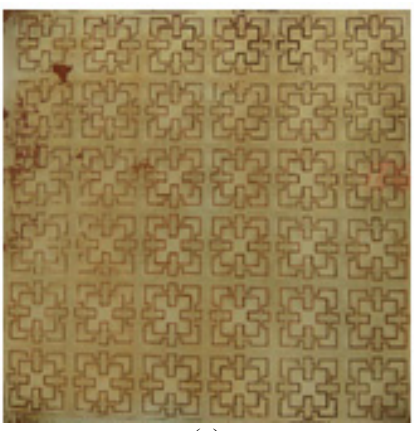

(a)

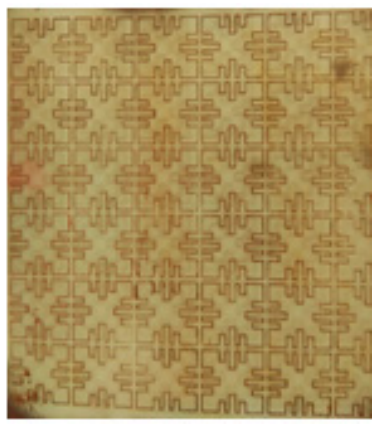

(b)
Fig. 8. Photograph of the fabricated prototype of $6 \times 6$ array of the proposed FSS: (a) Top view. (b) Bottom view.

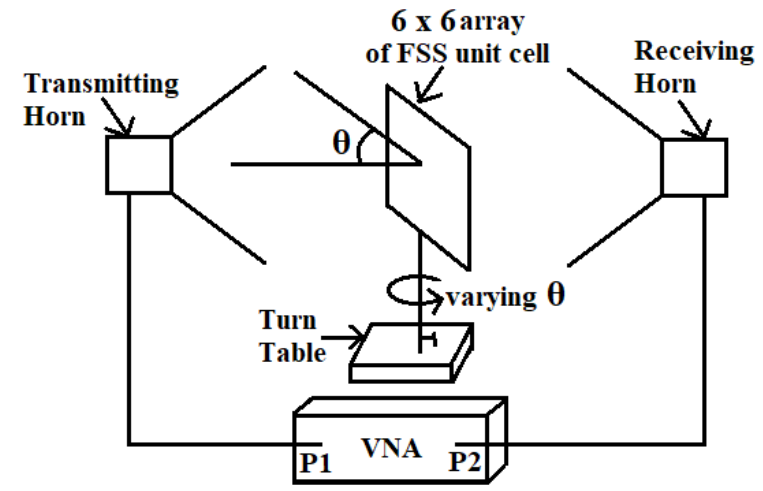

Fig. 9. Measurement set-up for the fabricated FSS.

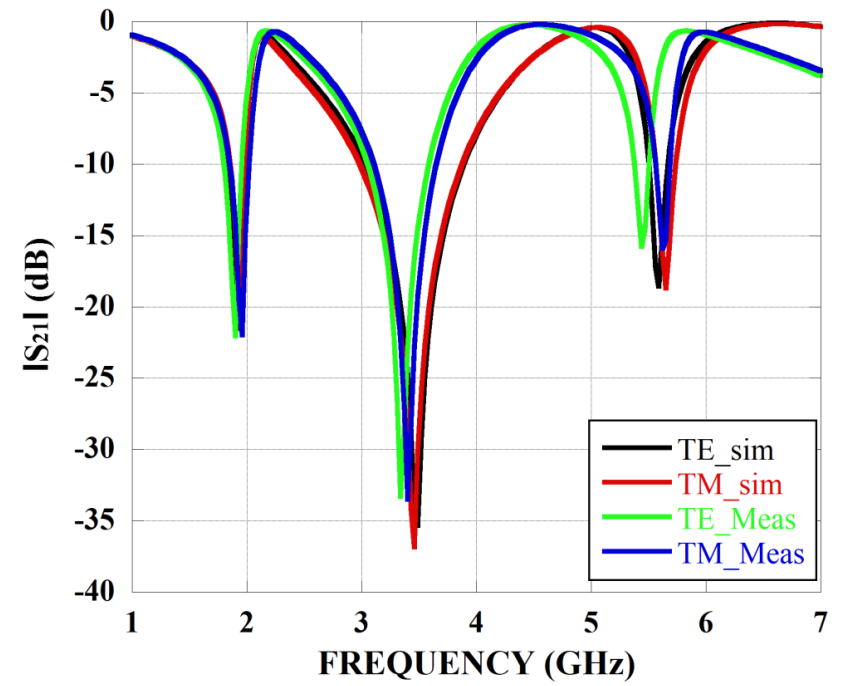

(a)

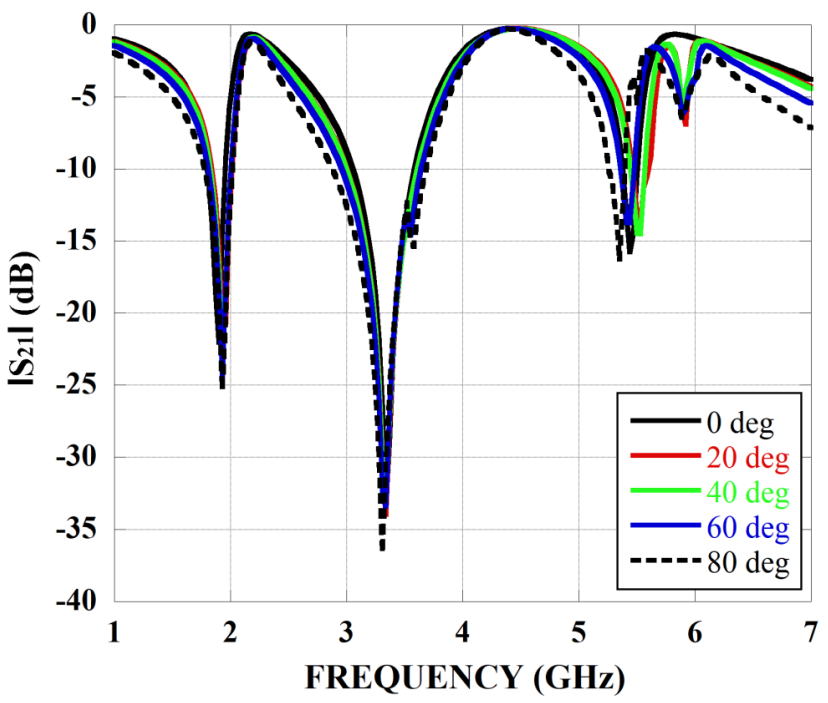

(b)

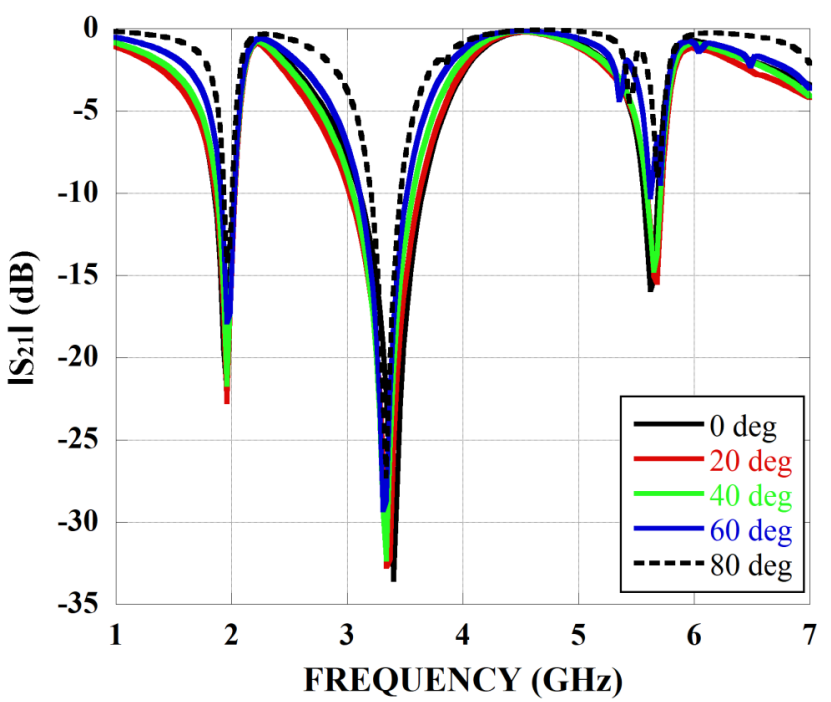

(c)

Fig. 10. Measured characteristics of the proposed FSS: (a) under normal incidence for both TE and TM modes; (b) under oblique incidence for TE mode; (c) under oblique incidence for TM mode. 
Figure 10(a) shows that simulated and measured results agree for both TE and TM modes under normal incidence. Figs. 10(b) and 10(c) illustrate the measured characteristics under oblique incidence which resemble closely with the simulated results given in Figs. 5(a) and 5(b) thus demonstrating the validity of the proposed FSS under oblique incidence. The slight error in measurement is due to fabrication error and finite array size of the FSS which causes edge diffraction.

\section{Conclusion}

A compact bandstop FSS has been proposed which has three metallic convoluted loop structures generating three stopbands at GSM, WLAN and WiMAX frequencies. The mechanism of stopband generation has been analyzed using current distribution patterns and equivalent circuit modeling. The structure has similar transmission characteristics under both TE and TM modes of propagating EM waves. It is highly stable under oblique incidence up to $\pm 80^{\circ}$. Comparison with similar research has demonstrated that the proposed structure exhibits the best performance with respect to multiple stopbands and immunity to oblique incident angle thus indicating its suitability in practical wireless applications amidst complex multipath environment. A prototype of the proposed structure has been fabricated and measured to validate the simulated results.

\section{References}

[1] ZHAO, P., ZONG, Z., LI, B., et al. Miniaturised bandstop frequency selective surface based on quasi-lumped inductor and capacitor. Electronics Letters, 2017, vol. 53, no. 10, p. 642-644. DOI: $10.1049 / \mathrm{el} .2017 .0548$

[2] ZHANG, K., JIANG, W., GONG, S. Design of bandpass frequency selective surface absorber using LC resonators. IEEE Antennas and Wireless Propagation Letters, 2017, vol. 16, p. 2586-2589. DOI: 10.1109/LAWP.2017.2734918

[3] KERN, D. J., WERNER, D. H., MONORCHIO, A., et al. The design synthesis of multiband artificial magnetic conductors using high impedance frequency selective surfaces. IEEE Transactions on Antennas and Propagation, 2005, vol. 53, no. 1, p. 8-17. DOI: 10.1109/TAP 2004.840540

[4] ZHONG, T., ZHANG, H., WU, R., et al. A frequency selective surface with polarization rotation based on substrate integrated waveguide. Progress in Electromagnetics Research Letters, 2016, vol. 60, p. 121-125. DOI: 10.2528/PIERL16031502

[5] GHOSH, A., MITRA, A., DAS, S. Meander line-based low profile RIS with defected ground and its use in patch antenna miniaturization for wireless applications. Microwave and Optical Technology Letters, 2017, vol. 59, no. 3, p. 732-738. DOI: 10.1002/mop.30384

[6] BILAL, M., SALEEM, R., SHABBIR, T., et al. A novel miniaturized FSS based electromagnetic shield for SATCOM applications. Microwave and Optical Technology Letters, 2017, vol. 59, no. 9, p. 2107-2112. DOI: 10.1002/mop.30696

[7] LI, J., ZENG, Q., LIU, R., et al. A compact dual-band beamsweeping antenna based on active frequency selective surfaces.
IEEE Transactions on Antennas and Propagation, 2017, vol. 65, no. 4, p. 1542-1549. DOI: 10.1109/TAP.2017.2669719

[8] LIU, N., SHENG, X. J., FAN, J. J. A compact miniaturized frequency selective surface with stable resonant frequency. Progress in Electromagnetics Research Letters, 2016, vol. 62 , p. 17-22. DOI: 10.2528/PIERL16070608

[9] GHOSH, S., SRIVASTAVA, K. V. An angularly stable dual-band FSS with closely spaced resonances using miniaturized unit cell. IEEE Microwave and Wireless Components Letters, 2017, vol. 27, no. 3, p. 218-220. DOI: 10.1109/LMWC.2017.2661683

[10] CAN, S., KAPUSUZ, K. Y., YILMAZ, A. E. A dual-band polarization independent FSS having a transparent substrate for ISM and Wi-Fi shielding. Microwave and Optical Technology Letters, 2017, vol. 59, no. 9, p. 2249-2253. DOI: $10.1002 /$ mop.30715

[11] KHAJEVANDI, S., ORAIZI, H., POORDARAEE, M. Design of planar dual-bandstop FSS using square-loop-enclosing superformula curves. IEEE Antennas and Wireless Propagation Letters, 2018, vol. 17, no. 5, p. 731-734. DOI: 10.1109/LAWP.2018.2812698

[12] NASROLlAHI, H., YEGANEH, A. N., SEDIGHY, S. H., et al. Compact, dual polarized, mutliband frequency selective surface with wideband spurious rejection. Microwave and Optical Technology Letters, 2017, vol. 59, no. 4, p. 888-893. DOI: 10.1002/mop. 30420

[13] LIU, N., SHENG, X., ZHANG, C., et al. A miniaturized triband frequency selective surface based on convoluted design. IEEE Antennas and Wireless Propagation Letters, 2017, vol. 16 , p. 2384-2387. DOI: 10.1109/LAWP.2017.2719859

[14] POOJALI, J., RAY, S., PESALA, B., et al. Quad-band polarization-insensitive millimeter-wave frequency selective surface for remote sensing. IEEE Antennas and Wireless Propagation Letters, 2017, vol. 16, p. 1796-1799. DOI: 10.1109/LAWP.2017.2679204

[15] KARTAL, M., GOLEZANI, J. J., DOKEN, B. A triple band frequency selective surface design for GSM systems by utilizing a novel synthetic resonator. IEEE Transactions on Antennas and Propagation, 2017, vol. 65, no. 5, p. 2724-2727. DOI: 10.1109/TAP.2017.2670230

[16] KARTAL, M., DOKEN, B. Triple band frequency selective surface design for global system for mobile communication systems. IET Microwaves, Antennas and Propagation, 2016, vol. 10, no. 11, p. 1154-1158. DOI: 10.1049/iet-map.2016.0021

[17] POZAR, D. M. Microwave Engineering. 4th ed., rev. Hoboken, New Jersey: John Wiley \& Sons, 2012. ISBN: 978-81-265-4190-4

[18] YILMAZ, A. E, KUZUOGLU, M. Design of the square loop frequency selective surfaces with particle swarm optimization via the equivalent circuit model. Radioengineering, 2009, vol. 18, no. 2, p. 95-102. ISSN 1210-2512

\section{About the Authors ...}

Anumoy GHOSH was born in 1987. He received the B. Tech. degree in Electronics and Communication Engineering from Kalyani Govt. Engineering College of West Bengal University of Technology, India, in 2010. He has obtained the M.E. degree from the Bengal Engineering and Science University, Shibpur, India, in 2012 and Ph.D degree from the Indian Institute of Engineering Science and Technology, Shibpur, India, in 2018. Since 2014, he is associated with the Department of Electronics and Communication Engineering of National Institute of Technology, Mizoram, India as Assistant Professor. His current 
research interests include planar antennas, periodic structures and smart antennas.

Amartya BANERJEE was born in 1992. He received the B. Tech. degree in Electronics and Communication Engineering from St. Thomas' College of Engineering and Technology, Kolkata, India, in 2014. He obtained the M.E. degree from the Bengal Engineering and Science University, Shibpur, India, in 2016. Presently, he is pursuing his Ph.D degree in Jadavpur University, Kolkata. His current research interests include antenna engineering, circuit modeling and analysis and metamaterial structures.
Santanu DAS was born in 1968. He received the B.E. degree in Electronics and Telecommunication Engineering from the Bengal Engineering College of Calcutta University (India) in 1989 and M.E. degree in Microwave Engineering from Jadavpur University, Calcutta, in 1992. He obtained Ph.D. (Engineering) degree from Jadavpur University in 1998. He presently holds the position of Professor in the Indian Institute. of Engineering Science and Technology, Shibpur, India. His current research interests include microstrip circuits, FSS, antenna elements and arrays. He is a life member of the Institution of Engineers, India. 